\title{
Исследование глубоких энергетических уровней в солнечном элементе типа HIT
}

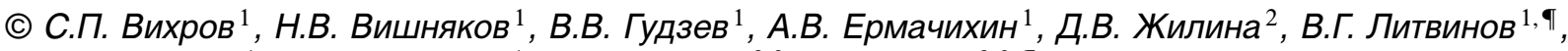 \\ А.Д. Маслов ${ }^{1}$, В.Г. Мишустин ${ }^{1}$, Е.И. Теруков ${ }^{2,3}$, А.С. Титов ${ }^{2,3,9}$ \\ ${ }^{1}$ Рязанский государственный радиотехнический университет, \\ 390005 Рязань, Россия \\ ${ }^{2}$ НТЦ тонкопленочных технологий в энергетике, \\ 194064 Санкт-Петербург, Россия \\ ${ }^{3}$ Физико-технический институт им. А.Ф. Иофффе Российской академии наук, \\ 194021 Санкт-Петербург, Россия \\ ฯ E-mail: vglit@yandex.ru, titovoz@gmail.com
}

(Получена 8 июня 2017 г. Принята к печати 19 июня 2017 г.)

\begin{abstract}
Представлены результаты исследования солнечного элемента типа $\mathrm{HIT} \mathrm{Ag} / \mathrm{ITO} / a-\mathrm{Si}: \mathrm{H}(p) / a-\mathrm{Si}: \mathrm{H}(i)$ / $c-\mathrm{Si}(n) / a-\mathrm{Si}: \mathrm{H}(i) / a-\mathrm{Si}: \mathrm{H}\left(n^{+}\right) / \mathrm{ITO} / \mathrm{Ag}$ методами вольт-фарадных характеристик и токовой релаксационной спектроскопии глубоких уровней. Исследованы температурная зависимость вольт-фарадных характеристик HIT-структуры и параметры глубоких энергетических уровней. Результаты комплексных исследований перечисленными методами были использованы для определения особенностей зонной диаграммы реальной НІТ-структуры.
\end{abstract}

DOI: 10.21883/FTP.2018.07.46053.8666

\section{1. Введение}

Солнечные элементы (СЭ) на основе структуры типа HIT (heterojunction with intrinsic thin layer) с использованием гетероперехода аморфного гидрогенизированного и монокристаллического кремния, $a-\mathrm{Si}: \mathrm{H} / c-\mathrm{Si}$, представляют большой интерес для теоретического и практического изучения. Их актуальность обусловлена низкой себестоимостью, невысокими температурами технологических процессов во время их получения, высоким значением эффективности фотоэлектрического преобразования и при этом высокой стабильностью характеристик $[1,2]$. Структуры на основе гетероперехода $a-\mathrm{Si}: \mathrm{H} / c-\mathrm{Si}$ часто используются в качестве модельных, так как. эта гетеропара достаточно хорошо изучена. Однако существует проблема понимания и контроля процессов рекомбинации носителей заряда на границах раздела слоев. На границах раздела слоев и в самих слоях аморфного кремния присутствуют энергетические состояния, которые могут проявлять себя подобно дефектам, образующим глубокие энергетические уровни (ГУ) в запрещенной зоне в кристаллическом полупроводнике. ГУ являются центрами рекомбинации для свободных носителей заряда и поэтому сильно влияют на коэффициент полезного действия прибора. Вследствие этого возникают трудности при моделировании параметров солнечных элементов. Введение в структуру тонкого буферного слоя на основе собственного аморфного кремния $a-\mathrm{Si}: \mathrm{H}(i)$ между слоями легированного $a$-Si : Н и кристаллической подложкой позволяет уменьшить плотность состояний на интерфейсах и снизить потери фотогенерированных носителей заряда на рекомбинацию.
Исследование электрических свойств гетеропереходов $a-\mathrm{Si}: \mathrm{H} / c-\mathrm{Si}$ достаточно успешно осуществляется методами спектроскопии адмиттанса, компьютерного моделирования, как описано в работе [3]. Однако между результатами компьютерного моделирования и результатами эксперимента наблюдается ряд противоречий. Моделирование характеристик чаще всего связано с подгонкой величин таких параметров реальной структуры, как плотность энергетических состояний, величин разрывов разрешенных энергетических зон, заложенных в ограниченную идеализированную физическую модель исследуемого объекта. Поэтому комплексное изучение НІТ-структуры необходимо для исследования электрических свойств, параметров ГУ, величин разрывов зон, уточнения параметров зонной диаграммы реальной структуры, что особенно необходимо при оптимизации конструкции СЭ для достижения целевых параметров и характеристик.

\section{2. Тестовые образцы и методика эксперимента}

В качестве объектов исследования для определения параметров глубоких центров и уточнения зонной диаграммы была выбрана HIT-структура $\mathrm{Ag} / \mathrm{ITO} / a-\mathrm{Si}: \mathrm{H}(p) /$ $a-\mathrm{Si}: \mathrm{H}(i) / c-\mathrm{Si}(n) / a-\mathrm{Si}: \mathrm{H}(i) / a-\mathrm{Si}: \mathrm{H}\left(n^{+}\right) / \mathrm{ITO} / \mathrm{Ag}$, схематически изображенная на рис. 1 . Толщина фотоактивного слоя кристаллического кремния составляла 180 мкм, слои $a-\mathrm{Si}: \mathrm{H}(p)$ и $a-\mathrm{Si}: \mathrm{H}(n)$ имели толщину 10 нм каждый, буферные слои $a-\mathrm{Si}: \mathrm{H}(i)$ толщиной 3 нм формировались между подложкой $c-\mathrm{Si}(n)$ и слоями $a-\mathrm{Si}: \mathrm{H}(p)$, $a-\mathrm{Si}: \mathrm{H}(n)$. Сверху и снизу структуры поверх слоев легированного аморфного гидрогенизированного кремния 


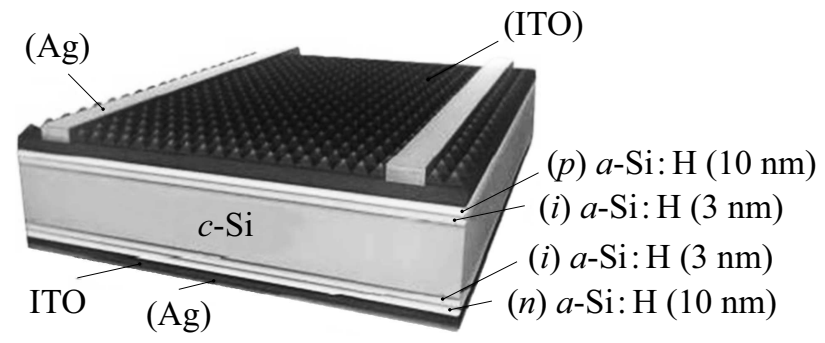

Рис. 1. Структура солнечного элемента НIT.

нанесены тонкие слои ITO (indium-tin oxide) толщиной 120 нм и сформированы омические серебряные контакты для проведения электрических измерений.

НІТ-структура выращена в НТЦ „Тонкопленочные технологии в энергетике“ Физико-технического института им. А.Ф. Иоффе РАН (Санкт-Петербург, Россия). Электрофизические измерения проведены в Региональном центре зондовой микроскопии коллективного пользования Рязанского государственного радиотехнического университета (Рязань, Россия).

Выращенные структуры были изучены методами токовой релаксационной спектроскопии глубоких уровней (I-DLTS) $[4,5]$ и методом вольт-фарадных характеристик $(C-V$-характеристик). Измерительный комплекс был адаптирован для изучения диодных структур солнечных элементов с относительно большой площадью барьерных контактов и большой величиной электрической барьерной емкости [6].

$C-V$-характеристики были измерены в температурном диапазоне $150-320 \mathrm{~K}$ для ряда фиксированных частот в диапазоне 100 Гц-10кГц с использованием LCR-метра Agilent E4980A. Измерительная ячейка выполнена на базе гелиевого криостата замкнутого типа Janis CCS400/204N.

\section{3. Результаты эксперимента и обсуждение}

Для построения зонной диаграммы (рис. 2) $p-i-n-$ фрагмента НІТ-структуры была использована модель Андерсона. Положение уровня Ферми $E_{F}$ относительно дна зоны проводимости $E_{c}$ в слое монокристаллического кремния $c-\mathrm{Si}(n)$ было рассчитано на основе определенной из $C-V$-характеристик величины концентрации свободных электронов.

$C-V$-характеристики были получены при напряжениях постоянного смещения $V$ от -2 до $1 \mathrm{~B}$ с шагом $20 \mathrm{MB}$, при амплитуде напряжения тестового измерительного сигнала $30 \mathrm{MB}$. На рис. 3 показаны измеренные $C-V$-характеристики в координатах $1 / C^{2}=f(V)$ при положительном смещении. Следует отметить, что линейная часть зависимостей простирается вплоть до обратного напряжения -2 В (на рис. 3 не показано).
Таблица 1. Величина концентрации свободных носителей заряда, определенная из $C-V$-измерений

\begin{tabular}{c|c|c|c|c}
\hline $\begin{array}{c}\text { Частота } \\
\text { измерительного } \\
\text { сигнала }\end{array}$ & \multicolumn{4}{|c}{ Концентрация, $10^{15} \mathrm{~cm}^{-3}$} \\
\cline { 2 - 5 } & $150 \mathrm{~K}$ & $200 \mathrm{~K}$ & $250 \mathrm{~K}$ & $300, \mathrm{~K}$ \\
\hline 100 Гц & 2.5 & 2.3 & 2.5 & 8.2 \\
10 КГц & 2.2 & 2.1 & 2.2 & 2.5
\end{tabular}

При прямом напряжении смещения $>0.4 \mathrm{~B}$ начинается инжекция свободных носителей заряда, что приводит к резкому росту электрической емкости за счет добавления диффузионной составляющей емкости. Концентрация свободных носителей на границе области пространственного заряда в слое $c-\operatorname{Si}(n)$ была оценена по наклону линейной части $C-V$-характеристик (рис. 3) с использованием известного приближения обедненного слоя для резкого перехода [7].

При низких температурах концентрация свободных носителей заряда, полученная из вольт-фарадных измерений, практически не зависит от частоты тестового сигнала (табл. 1). Свободными носителями заряда являются в данном случае свободные электроны в $c-\operatorname{Si}(n)$. При температуре $300 \mathrm{~K}$ и низких частотах наклон $C-V$-характеристики уменьшается и определяемая по углу этого наклона концентрация свободных носителей заряда в 3.3 раза больше, чем на высоких частотах. В диапазоне частот от 10 кГц до $1 \mathrm{MГц} \mathrm{изме-}$ ренные $C-V$-характеристики практически не отличались друг от друга. Электрическая емкость НIT-структуры определяется зарядом локализованных энергетических состояний вблизи уровня Ферми в обедненной области слоев $a-\mathrm{Si}: \mathrm{H}(i), a-\mathrm{Si}: \mathrm{H}(p)$ и на границе раздела $a-\mathrm{Si}: \mathrm{H}(i) / c-\mathrm{Si}(n)$. По мере роста температуры увеличивается скорость эмиссии носителей заряда с состояний в

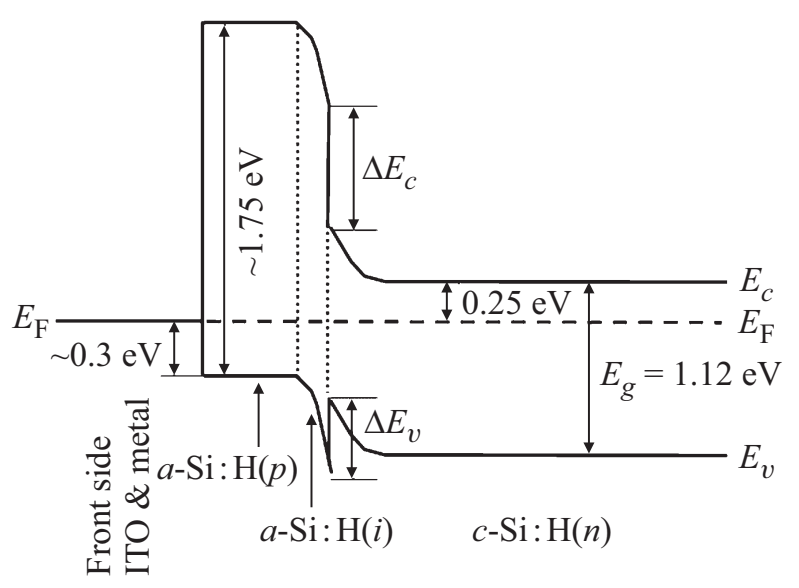

Рис. 2. Фрагмент зонной диаграммы НІТ-структуры. $E_{g}-$ ширина запрещенной зоны, $\Delta E_{c}, \Delta E_{v}$ - разрывы зон проводимости и валентной на гетерогранице. 

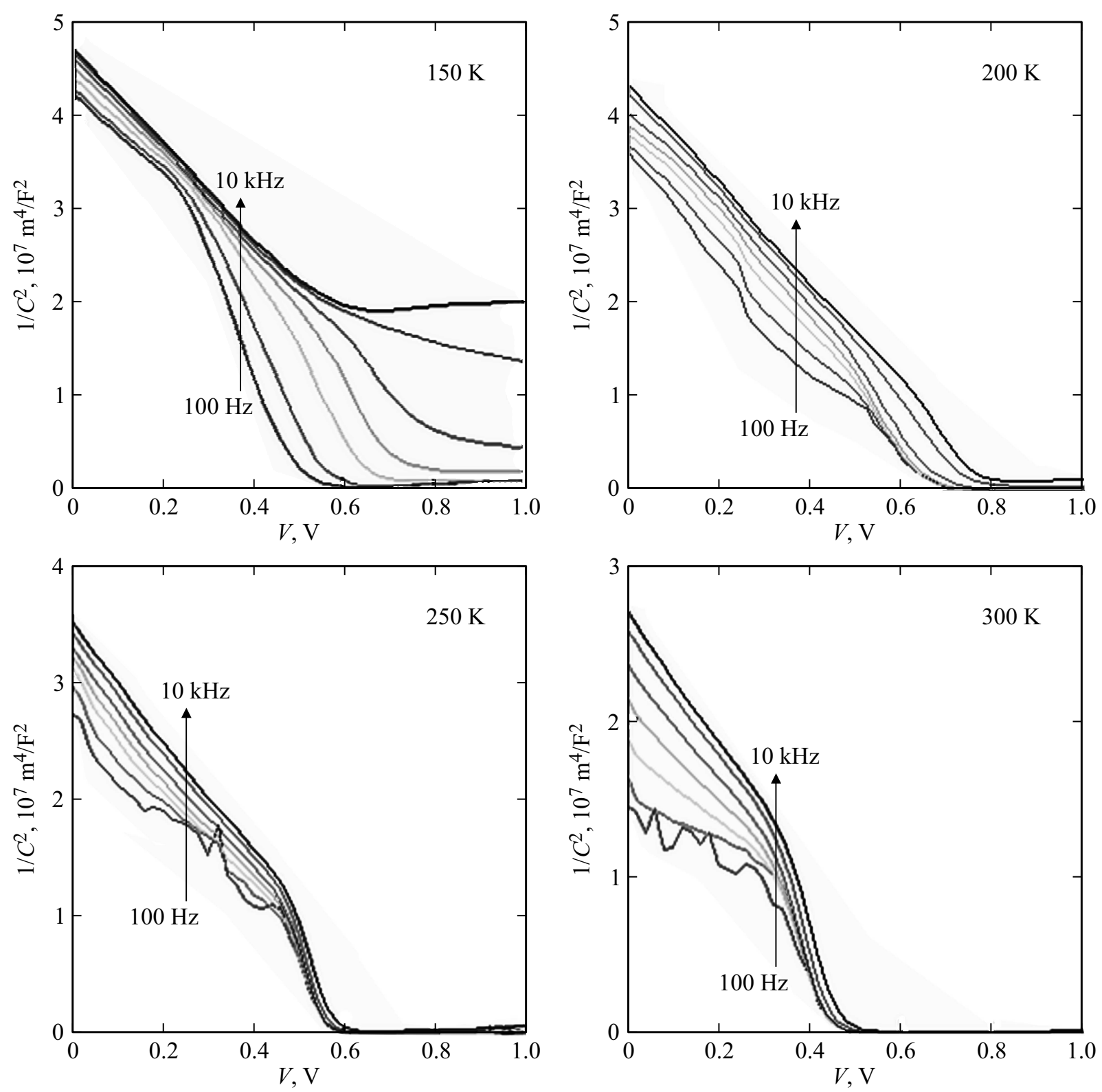

Рис. 3. $C-V$-характеристики НІТ-структуры при разных температурах, измеренные на частотах $100,200,500$ Гц, $1,2,5,10$ кГц.

$a-\mathrm{Si}: \mathrm{H}$. В случае, когда скорость эмиссии сопоставима с частотой изменения внешнего электрического поля, заряд локализованных состояний вносит вклад в барьерную емкость структуры.

На рис. 4 представлены зависимости емкости от температуры, $C(T)$, измеренные при разных частотах. Наличие ступени на характеристиках $C(T)$, измеренных в диапазоне частот от 100 Гц до 1 кГц в области температур 250-300 K, связано с вкладом локализованных состояний вблизи уровня Ферми в эмиттере $a-\mathrm{Si}: \mathrm{H}(p)[8]$. По температурному положению ступени можно определить энергетический промежуток между положением потолка валентной зоны $E_{v}$ и уровнем Ферми $E_{F}$ в слое $a-\mathrm{Si}: \mathrm{H}(p)$. Наблюдаемые ступени характеризуются незначительным и протяженным на- клоном, поэтому результат расчета указанной энергетической характеристики будет ненадежным. По этой причине HIT-структура также была исследована методом I-DLTS.

I-DLTS-спектры были измерены при амплитуде заполняющего импульса напряжения $U_{f}=0 \mathrm{~B}$ и амплитуде импульса опустошения $U_{r}=-1 \mathrm{~B}$. Длительность заполняющего импульса составляла 1 мс. На рис. 5 показаны полученные I-DLTS-спектры HIT-структуры. В каждом спектре наблюдается по три пика. Величины энергии активации были получены по углу наклона прямых Аррениуса согласно классическим представлениям о температурной зависимости скорости эмиссии носителей заряда с ГУ [4,5]. I-DLTS-спектры измерены при значениях времени релаксации $\tau$ в диапазоне от 46 мкс 
Таблица 2. Параметры ГУ, полученные из I-DLTS-спектров

\begin{tabular}{l|c|c|c}
\hline \multicolumn{1}{c|}{ ГУ } & $\mathrm{E} 1$ & $\mathrm{E} 2$ & $\mathrm{E} 3$ \\
\hline $\begin{array}{l}\text { Энергия } \\
\text { активации } \Delta E_{t}, \text { эВ }\end{array}$ & $0.07 \pm 0.02$ & $0.36 \pm 0.02$ & $0.72 \pm 0.03$ \\
\hline $\begin{array}{l}\text { Слоевая } \\
\text { концентрация } \\
N_{t} d, \mathrm{~cm}^{-2}\end{array}$ & $1.7 \cdot 10^{10}$ & $1.4 \cdot 10^{10}$ & $3.9 \cdot 10^{10}$
\end{tabular}

до 141 мкс. В табл. 2 приведены параметры обнаруженных ГУ. Все три ГУ имеют относительно высокие величины формально рассчитываемой концентрации в предположении о равномерном распределении глубоких центров, сопоставимые с концентрацией свободных электронов в $c-\mathrm{Si}(n)$. Мы полагаем, что обнаруженные пики в спектрах связаны с процессами эмиссии носителей заряда в слоях $a-\mathrm{Si}: \mathrm{H}(i)$ и $a-\mathrm{Si}: \mathrm{H}(p)$. ГУ $\mathrm{E} 1$ с наименьшей энергией активации 0.07 э обнаружен в области низких температур. Амплитуда пика Е1 не зависела от постоянной времени релаксации. Однако природа уровня Е1 требует дальнейшего изучения и до конца не ясна.

Пик Е2 может быть связан с процессом эмиссии дырок с локализованных состояний в запрещенной зоне вблизи уровня Ферми в обедненной области слоя $a$ - $\mathrm{Si}: \mathrm{H}(p)$. В работе [3] из $C-V$-измерений было обнаружено, что при температурах вблизи $200 \mathrm{~K}$ и выше происходит термическая активация проводимости в $a$-Si : Н. Соответственно пик Е2 был обнаружен в диапазоне температур 200-250 К. Процесс эмиссии дырок с локализованных состояний вблизи уровня Ферми соответствует активации транспорта в слое $a-\mathrm{Si}: \mathrm{H}(p)$.

ГУ Е3 с энергией активации $\Delta E_{t}=0.72$ эВ соответствует пик в спектре с наибольшей амплитудой. Амплитуда пика Е3 не изменялась при изменении амплитуды импульса напряжения опустошения от -0.5 до -3 В. Это говорит о том, что глубокие центры, или состояния, соответствующие пику Е3, распределены неравномерно и локализованы вблизи границы раздела $a-\mathrm{Si}: \mathrm{H}(i) / c-\mathrm{Si}(n)$. Поскольку было обнаружено неравномерное распределение концентрации ГУ, то по этой причине рассчитывалась слоевая концентрация $N_{t} d$ $\left(N_{t}\right.$ - концентрация глубоких центров, участвующих в перезарядке носителями заряда, $d-$ ширина области пространственного заряда, в которой происходит эмиссия носителей заряда с ГУ).

Амплитуда пика Е3 сильно зависит от величины времени релаксации. Из рис. 5 видно, как амплитуда пика Е3 уменьшилась в 2.5 раза при изменении времени релаксации от 141 до 46 мкс. Это может быть связано c перезарядкой энергетических состояний на границе раздела гетероперехода $a-\mathrm{Si}: \mathrm{H}(i) / c-\mathrm{Si}(n)$ и локализованных состояний вблизи уровня Ферми в тонком слое собственного $a$-Si : H (рис. 2). Положение уровня Ферми практически не меняется при приложении обратного напряжения, так как его положение зафиксировано энергетическими состояниями. При приложении обратного напряжения к НIT-структуре квазиуровень Ферми в $c-\mathrm{Si}(n)$ смещается ниже его положения в $a-\mathrm{Si}: \mathrm{H}$, что приводит к эмиссии электронов в зону проводимости.

Таким образом, энергетический зазор между уровнем Ферми и потолком валентной зоны в $a-\mathrm{Si}: \mathrm{H}(p)$ составляет 0.36 эВ. Разница между уровнями Ферми на границе раздела $a-\mathrm{Si}: \mathrm{H}(i) / c-\mathrm{Si}(n)$ и зоной проводимости вблизи

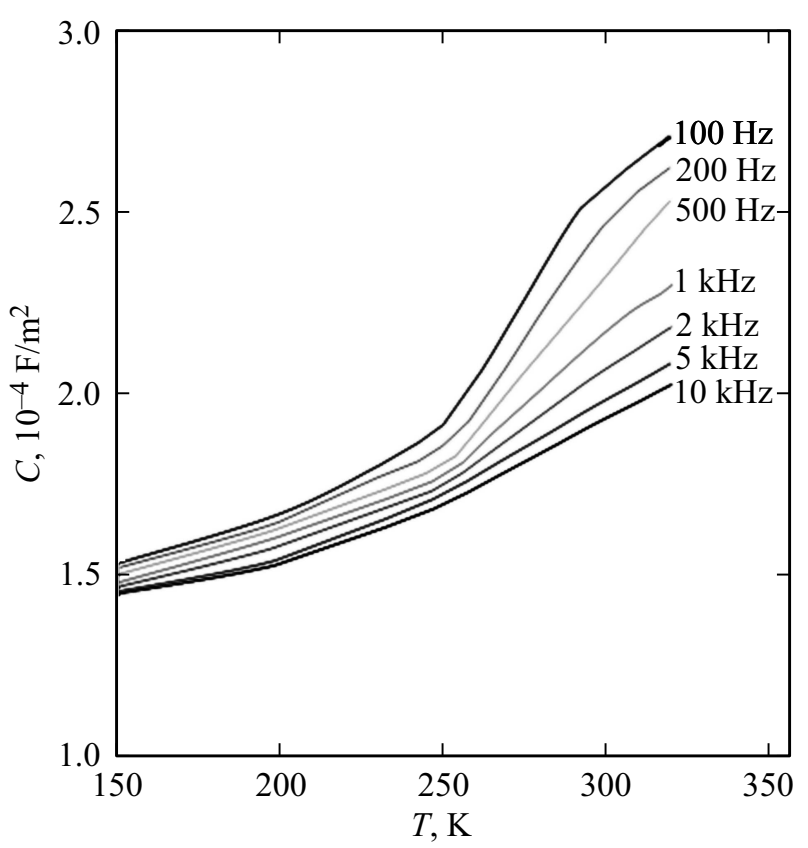

Рис. 4. Температурные зависимости емкости НІТ-структуры при смещении 0 В и разных частотах.

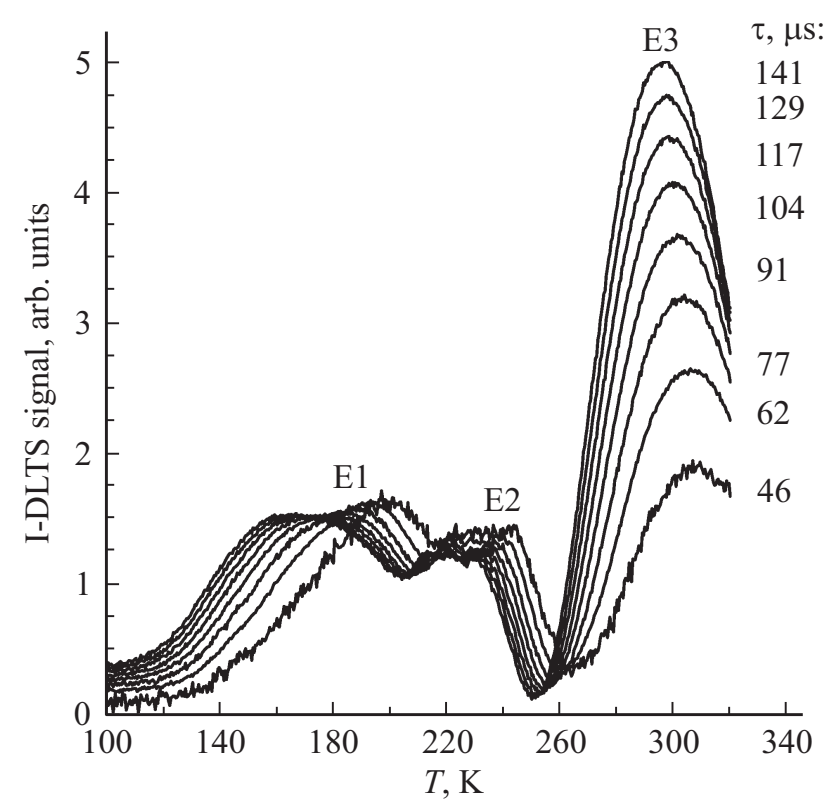

Рис. 5. I-DLTS-спектры HIT-структуры, полученные с использованием весовой функции „lock-in“ для разных величин постоянной времени релаксации $\tau$. 
границы раздела со слоем $c-\operatorname{Si}(n)$ равна 0.72 эВ при комнатной температуре.

\section{4. Заключение}

Представлены результаты комплексного исследования барьерной НIT-структуры $\mathrm{Ag} / \mathrm{ITO} / a-\mathrm{Si}: \mathrm{H}(p) /$ $a$-Si : $\mathrm{H}(i) / c-\mathrm{Si}(n) / a-\mathrm{Si}: \mathrm{H}(i) / a-\mathrm{Si}: \mathrm{H}\left(n^{+}\right) / \mathrm{ITO} / \mathrm{Ag}$ методом $C-V$-характеристик при разных температурах и частотах, а также методом I-DLTS. По результатам исследования уточнены параметры зонной диаграммы реальной НIT-структуры. Уточнены положения уровней Ферми в слое $a-\mathrm{Si}: \mathrm{H}(p)$ и на границе раздела $a-\mathrm{Si}: \mathrm{H}(i) / c-\mathrm{Si}(n)$.

Работа выполнена при финансовой поддержке Министерства образования и науки РФ на оборудовании Регионального центра зондовой микроскопии коллективного пользования Рязанского государственного радиотехнического университета.

\section{Список литературы}

[1] K. Wakisaka, M. Taguchi, T. Sawada, M. Tanaka, T. Matsuyama, T. Matsuoka, S. Tsuda, S. Nakano, Y. Kishi, Y. Kuwano. Proc. 22nd Photovoltaic Specialists Conf. (Las Vegas, 1991) p. 887.

[2] H. Sakata, Y. Tsunomura, H. Inoue, S. Taira, T. Baba, H. Kanno, T. Kinoshita, M. Taguchi, E. Maruyama. $R \& D$ progress of next-generation very thin HIT solar cells. 25th Eur. Photovoltaic Solar Energy Conf. Exh. (Valencia, 2010) p. 1102.

[3] A.S. Gudovskikh, J.P. Kleider, A. Froitzheim, W. Fuhs, E.I. Terukov. Thin Sol. Films, 451-452, 345 (2004).

[4] D.V. Lang. J. Appl. Phys., 45, 3023 (1974).

[5] J.A. Borsuk, R.M. Swanson. IEEE Trans. Electron Dev., ED-27, 2217 (1980).

[6] V.G. Litvinov, N.V. Vishnyakov, V.V. Gudzev, V.G. Mishustin, S.M. Karabanov, S.P. Vikhrov, A.S. Karabanov. Power Electronics and Renewable Energy Conversion USB, Proc. IEEE Intern. Conf. Industrial Technology (2015).

[7] S.M. Sze, Kwok K. Ng. Physics of semiconductor devices (John Wiley\&Sons, N.Y., 2006).

[8] A.S. Gudovskikh, J.P. Kleider, E.I. Terukov. Semiconductors, 39, 904 (2005).

Редактор Л.В. Шаронова

\section{Investigation of deep levels in solar cell structure based on HIT}

S.P. Vikhrov' ${ }^{1}$, N.V. Vishnyakov' ${ }^{1}$, V.V. Gudzev', A.V. Ermachikhin ${ }^{1}$, D.V. Shilina ${ }^{2}$, V.G. Litvinov ${ }^{1}$, A.D. Maslov' ${ }^{\text {, V.G. Mishustin }}{ }^{1}$, E.I. Terukov ${ }^{2,3}$, A.S. Titov ${ }^{2,3}$

\author{
${ }^{1}$ Ryazan State Radio Engineering University, \\ 390005 Ryazan, Russia \\ 2 loffe Institute, \\ 194021 St. Petersburg, Russia. \\ ${ }^{3}$ R\&D Center of Thin Film Technologies in Energetics \\ under loffe Institute LLC, \\ 194064 St. Petersburg, Russia
}

\begin{abstract}
The results of investigation of deep energy levels in barrier structures based on the HIT structure $\mathrm{Ag} / \mathrm{ITO} / a-\mathrm{Si}: \mathrm{H}(p)$ / $a$-Si : $\mathrm{H}(i) / c-\mathrm{Si}(n) / a-\mathrm{Si}: \mathrm{H}(i) / a-\mathrm{Si}: \mathrm{H}\left(n^{+}\right) / \mathrm{ITO} / \mathrm{Ag}$, designed to create the solar cells, are presented. The temperature dependences of the voltage-capacitance characteristics of HIT structures and the parameters of deep energy levels are studied. According to the research the model of energy band diagram of a HIT structure $\mathrm{Ag} / \mathrm{ITO} / s-\mathrm{Si}: \mathrm{H}(p) / a-\mathrm{Si}: \mathrm{H}(i) / c-\mathrm{Si}(n) / a-\mathrm{Si}: \mathrm{H}(i) / a-\mathrm{Si}: \mathrm{H}\left(n^{+}\right) /$ $\mathrm{ITO} / \mathrm{Ag}$ is developed.
\end{abstract}

